\title{
An Improved Robust Adaptive Beamforming Algorithm Based on the Conformal Array
}

\author{
Zhili Wang, Yuwen Wang, Zhenzhen Li \\ School of Astonautics and Aeronautics, UESTC Chengdu, China \\ gnaw014@163.com, wangyw@uestc.edu.cn, 742883363@qq.com
}

Keywords: Diagonal loading, Conformal array, Adaptive beamforming

\begin{abstract}
Diagonal loading algorithm is an effective method to improve the performance of beamforming and reduce the sidelobe level .But there is not have a theoretical method to reasonably determine the size of the diagonal loading value. This paper presents an improved adaptive beamforming algorithm that can effectively determine the diagonal loading value. Applied the algorithm in the conformal array models, Compared to the other two diagonal loading algorithms, The simulation results demonstrate its effectiveness.
\end{abstract}

\section{Introduction}

As the basis for array signal processing, Adaptive beamforming is widely used in radar, sonar, microphone array language processing, medical imaging, wireless communications and other fields. In order to improve the robustness of adaptive and combined theory algorithm with project practice, Scholars have proposed a series of adaptive beamforming algorithms such as Characteristics of interference cancellation[1], LCMV algorithm[2], Diagonal loading algorithm and Subspace beamforming algorithm[3,4].

Domestic and foreign scholars have made many improvements in the algorithms that mentioned above. Fixed diagonal loading algorithm is not flexible enough. The Loading level can not adjust its coefficient values adaptive according to the change of actual sample data. Taking into account the sampling covariance matrix can not figure out the actual covariance matrix precisely, To relieve the impact on the SINR(Signal to Interference and Noise Ratio)and the pattern function, Generalized linear combination(GLC) of adaptive beamforming algorithm[5] inject the white noise into sample covariance to increase the estimation accuracy of the covariance matrix. Then, it can enhance the performance of adaptive beamforming algorithm. However, its performance is still not the best. In addition, the analysis of conformal array is more complex and most of the studies are for the ordinary planar array.

In order to further improve the performance of adaptive beamforming in conformal array,this paper give out the analysis of pattern function with conformal array and put forward an improved adaptive beamforming algorithms that can effectively determine the diagonal loading value. The simulation results proved its effectiveness.

\section{Content}

Reception model of conformal array.

Conformal array model shown in Figure 1,its pattern function can be expressed as:

$$
F(\phi, \theta)=\sum_{n=1}^{N}\left[w_{n}^{*} f_{n}(\phi, \theta) e^{-j k p_{n} \cdot u}\right] .
$$




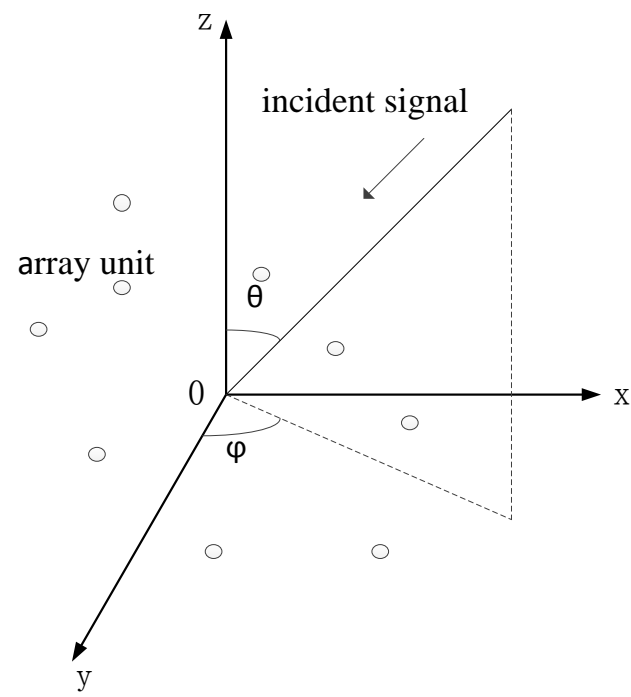

Fig. 1 reception model of conformal array

$p_{n}$ represents the coordinate of array unit and $p_{n}=\left[x_{n}, y_{n}, z_{n}\right], \mathrm{u}$ is the incident signal of unit direction vector and $u=-[\sin \theta \cos \varphi, \sin \theta \sin \varphi, \cos \theta], w_{n}$ is the complex weighting vector of the $\mathrm{N}$-th array unit and its radiation pattern vector shown by $f_{n}(\phi, \theta)$.Each conformal array unit of polar pattern are greatly influenced by radiation point and carrier curvature[6].these array units have different local coordinate system. Then, in this model, $f_{n}(\phi, \theta)$ can not be the same as plane array that can be extracted as a common factor. Pattern product theorem is no longer applicable. we can establish the conformal cylindrical array models as follows(Fig.2 and Fig.3).

If $m$ shows the layers which the array unit lie in, $n$ means the number of array unit that arranged with anti-clowise direction. we can give the expression of each array unit in $3 \mathrm{D}$ rectangular coordinate as follow:

$$
\left\{\begin{array}{l}
x_{m n}=R \cos \left((n-1) \beta-\theta_{0}\right)=R \cos (n \beta-(M+1) \beta / 2) \\
y_{m n}=R \sin \left((n-1) \beta-\theta_{0}\right)=R \sin (n \beta-(M+1) \beta / 2) \\
z_{m n}=(m-1) d-z_{0}=m d-(N+1) d / 2
\end{array}\right.
$$

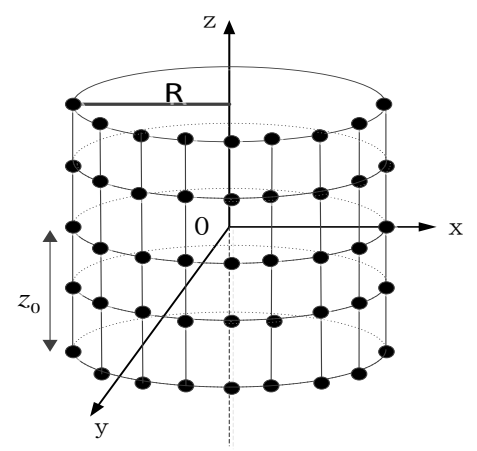

Fig.2. conformal cylindrical array model

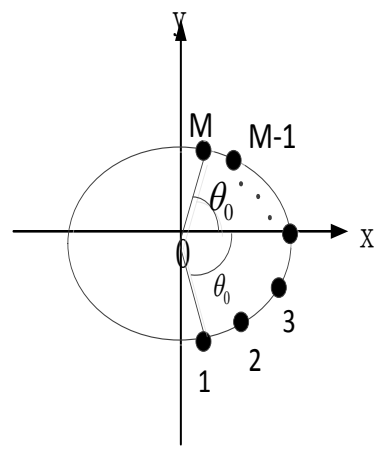

Fig.3 X-Y plane schematic

Among them, $\beta=2 \theta_{0} /(M-1), d=2 z_{0} /(N-1)$.Conformal array pattern function can be decomposed into two parts that expressed azimuth and elevation. Expression as follow:

$F(\phi, \theta)=F_{\phi}(\phi, \theta) \mathrm{u}_{\phi}+F_{\theta}(\phi, \theta) u_{\theta}$.

$F_{\phi}(\phi, \theta)$ is azimuth component and $F_{\theta}(\phi, \theta)$ is elevation component. There expression as follow: 


$$
\left\{\begin{array}{l}
F_{\phi}(\phi, \theta)=\sum_{n=1}^{N} w_{n \mid \phi} f_{n \mid \phi}(\phi, \theta) e^{j k p_{n} \cdot u}=W_{\phi}^{T} A_{\phi}(\phi, \theta) \\
F_{\theta}(\phi, \theta)=\sum_{n=1}^{N} w_{n \mid \theta} f_{n \mid \theta}(\phi, \theta) e^{j k p_{n} \cdot u}=W_{\theta}^{T} A_{\theta}(\phi, \theta)
\end{array}\right.
$$

$w_{n \mid \theta}$ and $w_{n \mid \phi}$ are the Weights of the N-th array unit, $W_{\theta}=\left[w_{1 \mid \theta}, w_{2 \mid \theta}, \cdots, w_{N \mid \theta}\right]^{T}$, $W_{\phi}=\left[w_{1 \mid \phi}, w_{2 \mid \phi}, \cdots, w_{N \mid \phi}\right]^{T} \cdot A_{\phi}(\phi, \theta)$ and $A_{\theta}(\phi, \theta)$ are the signal steering vectors.

Expression as follow:

$$
\left\{\begin{array}{l}
A_{\theta}(\phi, \theta)=\left[f_{1 \mid \theta}(\phi, \theta) \cdot \mathrm{e}^{j k p_{1} \cdot u}, f_{2 \mid \theta}(\phi, \theta) \cdot \mathrm{e}^{j k p_{2} \cdot u}, \cdots, f_{N \mid \theta}(\phi, \theta) \cdot \mathrm{e}^{j k p_{N} \cdot u}\right]^{T} \\
A_{\phi}(\phi, \theta)=\left[f_{1 \mid \phi}(\phi, \theta) \cdot \mathrm{e}^{j k p_{1} \cdot u}, f_{2 \mid \phi}(\phi, \theta) \cdot \mathrm{e}^{j k p_{2} \cdot u}, \cdots, f_{N \mid \phi}(\phi, \theta) \cdot \mathrm{e}^{j k p_{N} \cdot u}\right]^{T}
\end{array}\right.
$$

\section{Robust diagonal loading adaptive beamforming algorithm}

This algorithm is modeled by a optimization problem as follow:

$$
\left\{\begin{array}{c}
\min _{w} w^{H}(\tilde{R}+\delta I) w \\
\text { s.t. } \quad w^{H} a_{0}=1
\end{array}\right.
$$

Minimum variance distortion response beamformer can get the consequence as follow:

$$
w_{0}=\frac{(\tilde{R}+\delta I)^{-1} a_{0}}{a_{0}^{H}(\tilde{R}+\delta I)^{-1} a_{0}} .
$$

I is identity matrix and the $\delta$ is loading level. There is not have a theoretical method to reasonably determine the size of the diagonal loading value at present. This paper presents an improved adaptive beamforming algorithm that can effectively determine the diagonal loading value.

Disaggregated the data covariance matrix $\tilde{R}$ :

$$
\tilde{R}=U \Lambda U^{H}=\sum_{i=1}^{M} \lambda_{i} t_{i} t_{i}^{H}
$$

$U=\left[t_{1}, t_{2} \cdots t_{M}\right]$ show eigenvectors, $\Lambda=\operatorname{diag}\left\{\lambda_{1}, \lambda_{2}, \cdots, \lambda_{M}\right\}$ is the diagonal matrix of eigenvalues and sorted in descending order. Let us suppose the number of signals is $K$ that include the desired and interfering signals. The last $\mathrm{M}-\mathrm{K}$ represent the eigenvalues of noise signals.so, the data covariance matrix $\tilde{R}$ can be given by:

$$
\tilde{R}=A \Lambda_{s} A^{H}+\sigma_{n}^{2} I \text {. }
$$

A is steering vector. Noise power is $\sigma_{n}^{2} \cdot \Lambda_{s}=\operatorname{diag}\left\{\sigma_{1}^{2}, \sigma_{2}^{2}, \cdots, \sigma_{K}^{2}\right\}$ is the signal power of diagonal matrix. Usually, there are errors with $\tilde{R}$. The diagonal loading covariance matrix shown as:

$$
R_{l}=R+\mu D+\delta I .
$$

$\mathrm{R}$ is the real covariance matrix. $\mathrm{D}$ is random matrix with mean 0 and variance 1 . If $\mu\|D\| \leq\|R+\delta I\|, \quad R_{l}^{-1}$ is approximated at:

$$
R_{l}^{-1}=(R+\delta I)^{-1}\left\{I-\frac{\mu}{\delta+\sigma_{n}^{2}} D\left[I-A\left(A^{H} A+\left(\delta+\sigma_{n}^{2}\right) \Lambda_{s}^{-1}\right)^{-1} A^{H}\right]\right\} .
$$

If $(R+\delta I)^{-1}$ approximately equal to $\mathrm{R}$, The value of $\delta$ is

$\delta<R(i, i), i=1,2, \cdots, M$.

In order to get better performance of beam former, there must be:

$\frac{\mu}{\delta+\sigma_{n}^{2}}<1$.

Because of $\delta>0, \sigma_{n}^{2} \rightarrow 0$, we can get: 
$\delta \geq \mu$.

We can replace $R(i, i)$ with the average of sampling covariance matrix $\tilde{R}$ :

$R(i, i)=\operatorname{tr}(\tilde{R}) / M$.

$\operatorname{tr}(\cdot)$ means the trace of matrix.The estimate of $\mu$ can be the variance-covariance matrix of diagonal elements as follow:

$\mu=\operatorname{std}(\operatorname{diag}(\tilde{R}))$.

$\operatorname{diag}(\cdot)$ is the diagonal elements of matrix.std $(\cdot)$ is the standard deviation.Then,we can get the rang of diagonal loading value:

$\operatorname{std}(\operatorname{diag}(\tilde{R})) \leq \delta<\operatorname{tr}(\tilde{R}) / M$.

In this way, we choose the value of $\mu$ as:

$\delta=\operatorname{std}(\operatorname{diag}(\tilde{R}))$.

\section{Simulation results}

Uniform linear array(ULA).

Simulation condition:This simulation select ULA of 16 . Spacing of half a wavelength, $c=3 \times 10^{8} \mathrm{~m} / \mathrm{s}, f=3 \times 10^{9} \mathrm{~Hz}$.The desired signal direction angle is $0^{\circ}$.Two interference signals are $25^{\circ}$ and $50^{\circ}$.

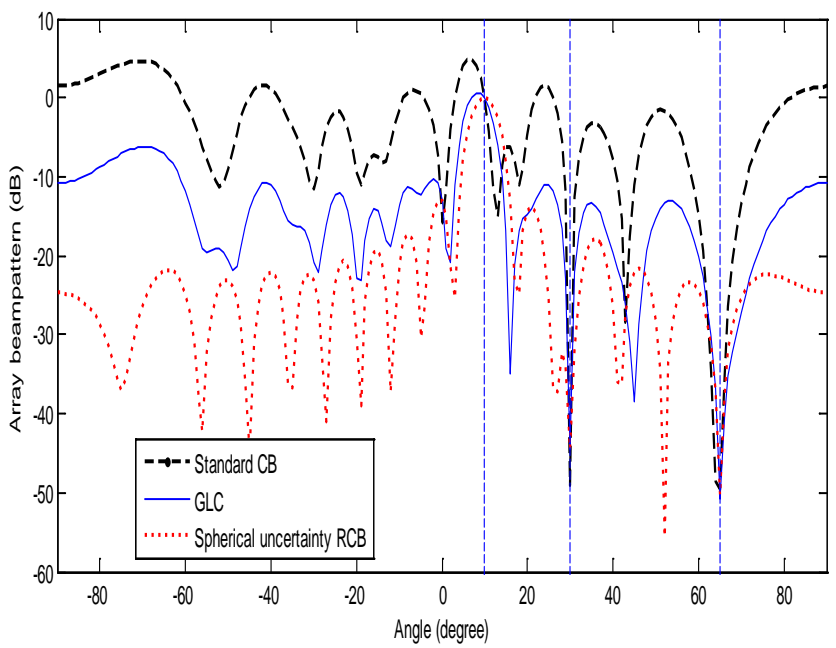

Fig.4 ULA of 3 kinds of robust beamformer

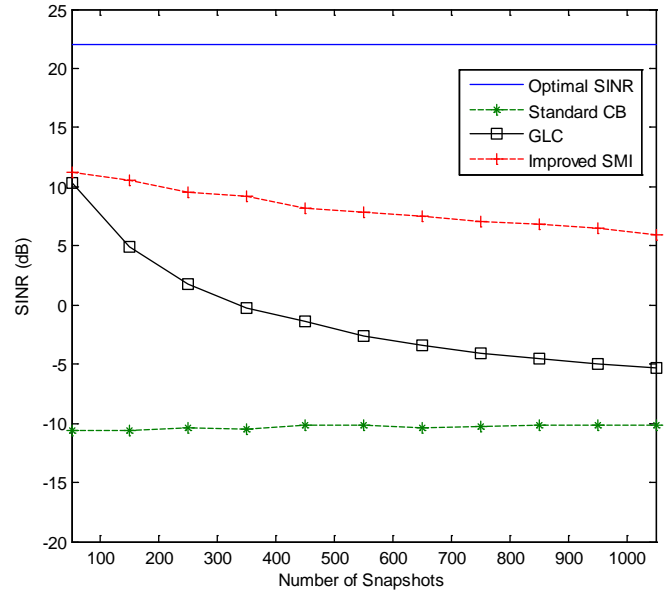

Fig.5 SINR with the change of snapshots

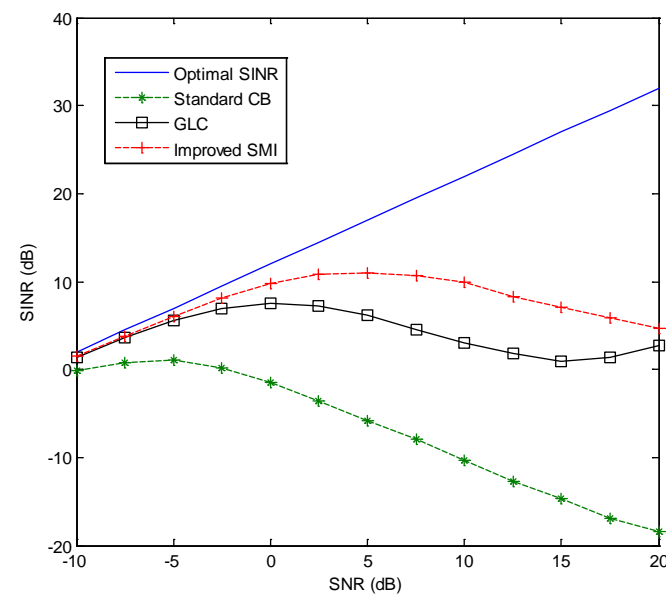

Fig.6 SINR with the change of SNR

From Fig.4, the conclusion is that the improved SMI can get lower sidelobe level than classic RCB algorithm of GLC. At the same time, it can also form deep nulls in the direction of 
interference. To reflect the the robustness of this algorithm, we select different snapshots and signal to noise ratio that can get different signal to interference noise ratios(SINR). The simulation results are Fig.5 and Fig.6.

From the comparison of the figure 5 and 6, we can conclude that the SINR will rise with the increasing of SNR and snapshots. When the snapshots exceed 500, SINR tends to stable. When the SNR is greater than 8dB,the SINR of improved SIM is bigger than GLC and Standard RCB. So, its performance is preferable.

\section{Conformal Array robust adaptive beamforming.}

Simulation condition: select a quarter of conformal cylindrical array of $6 \times 32,6$ layers, cylindrical radius $\mathrm{r}=0.2 \mathrm{~m}$, height of a circular cylinder $\mathrm{h}=0.3 \mathrm{~m}$, The desired signal direction angle is

$\left(0^{\circ},-3^{\circ}\right)$.Two interference signals are $\left(0^{\circ}, 25^{\circ}\right)$ and $\left(0^{\circ}, 40^{\circ}\right)$.The SNR of desired signal is $8 \mathrm{~dB}$. The SINR of interference signals are $20 \mathrm{~dB}$, The number of snapshots 500 .

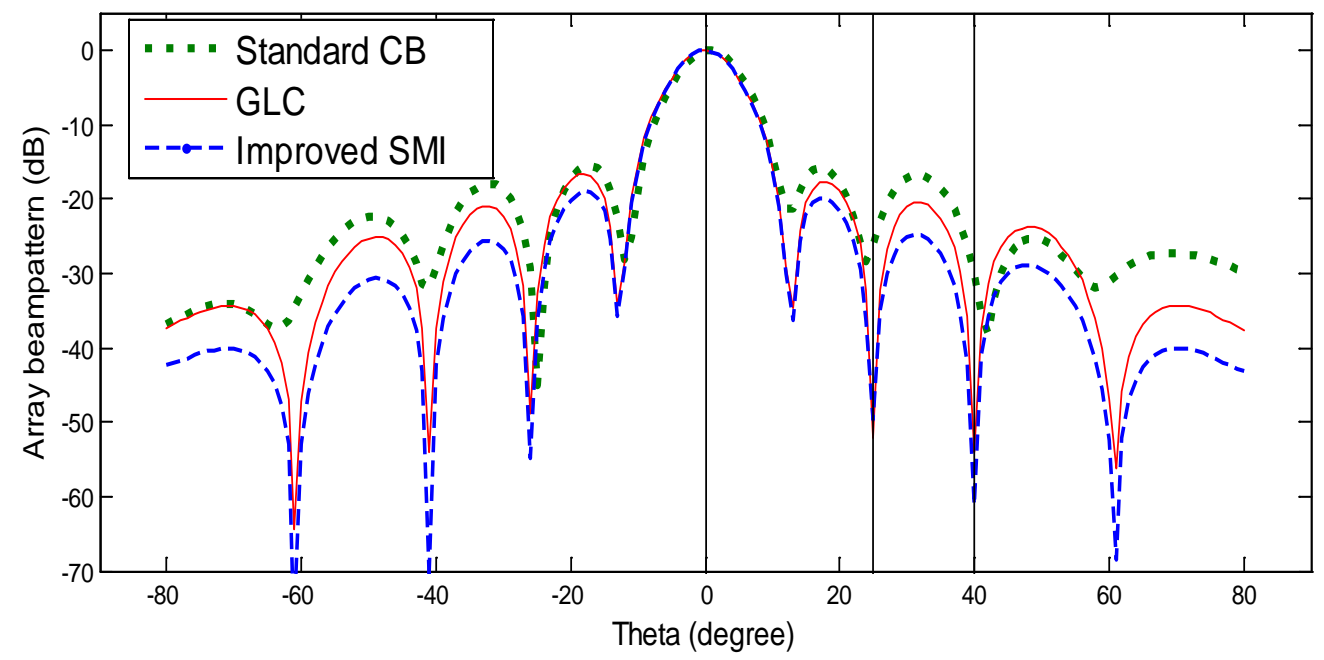

Fig.7 The elevation beam pattern of 3 kinds of robust beamformer

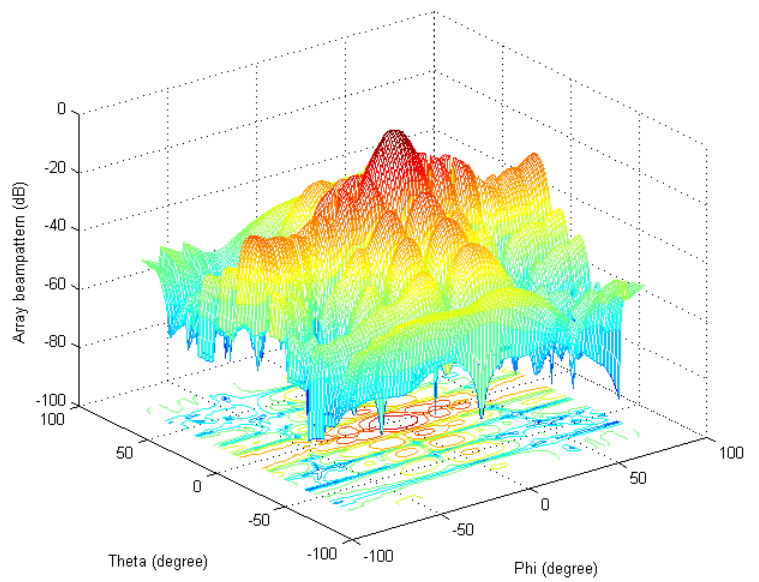

Fig.8 3D beam pattern of Improved SMI

From the figure 7 and the figure 8,the conclusion is that improved SMI can get lower sidelobe level. Meanwhile, it can form deep nulls in the direction of interference.

\section{Conclusion}

This paper discusses conformal array of robust adaptive beamforming algorithms.it put forward an improved SMI to enhance the robustness of the beam and reduce its sidelobes level. Simulation in the ULA and conformal array show that compared with the Standard RCB and the GLC, the improved SMI have good robustness and performance. 


\section{References}

[1] Haimovich A. The eigencanceler: adaptive radar by eigenanalysis methods[J]. IEEE Transactions on Aerospace \& Electronic Systems, 1996, 32(2):532 - 542.

[2] Tseng C. Minimum variance beamforming with phase-independent derivative constraints[J]. IEEE Transactions on Antennas \& Propagation, 1992, 40(3):285 - 294.

[3] Carlson B D. Covariance matrix estimation errors and diagonal loading in adaptive arrays[J]. IEEE Transactions on Aerospace Electronic Systems, 1988, 24(4):397 - 401.

[4] Feldman D D, Griffiths L J. A projection approach for robust adaptive beamforming[J]. IEEE Transactions on Signal Processing, 1994, 42(4):867 - 876.

[5] Du L, Li J, Stoica P. Fully Automatic Computation of Diagonal Loading Levels for Robust Adaptive Beamforming[J]. Aerospace \& Electronic Systems IEEE Transactions on, 2010, 46(1):449 - 458.

[6] Zi-sen Q, Ying G, Bu-hong W, et al. Joint DOA and Polarization Estimation Algorithm for Conformal Array Antenna[J]. Acta Electronica Sinica, 2012, 40(12):2562-2566. 